International Journal of Maternal and Child Health and AIDS (2020), Volume 9, Issue I, 22-33

\begin{tabular}{ll}
\hline & INTERNATIONAL JOURNAL of \\
& MATERNAL and CHILD HEALTH and AIDS \\
& ISSN 216I-864X (Online) \\
& ISSN 216I-8674 (Print) \\
IJMA Available online at www.mchandaids.org & DOI: 10.21 I 06/ijma.337 \\
\hline
\end{tabular}

\title{
ORIGINALARTICLE
}

\section{Social Determinants of Overweight and Obesity Among Children in the United States}

Zenab I.Yusuf, MBBS, MPH; ${ }^{\bowtie}$ Deepa Dongarwar, MS; ${ }^{2}$ Rafeek A Yusuf, MBBS, MPH, MS; ${ }^{3}$ Meishon Bell, MS; ${ }^{4}$ Toi Harris, MD; Hamisu M. Salihu, MD, $\mathrm{PhD}^{6}$

'Menninger Department of Psychiatry and Behavioral Sciences, Baylor College of Medicine, Houston, Texas, USA; Houston VA Health Services Research and Development Service Center for Innovations in Quality, Effectiveness and Safety, Michael E. DeBakey VA Medical Center, Houston; and VA South Central Mental Illness Research, Education and Clinical Center; ${ }^{2}$ Center of Excellence in Health Equity, Training and Research, Baylor College of Medicine, Houston, TX, USA; ${ }^{3}$ Department of Management, Policy, and Community Health, University of Texas Health Science Center at Houston, School of Public Health, Houston, TX, USA; ${ }^{4}$ Center of Excellence in Health Equity, Training and Research, Baylor College of Medicine, Houston, TX, USA; ${ }^{5}$ Menninger Department of Psychiatry and Behavioral Sciences, Baylor College of Medicine, Houston, Texas, USA; Department of Pediatrics, Baylor College of Medicine, Houston, TX, USA; and Center of Excellence in Health Equity, Training and Research, Baylor College of Medicine, Houston, TX, USA; ${ }^{6}$ FCM-Adminstartion Research, Baylor College of Medicine, Houston, TX, USA; Department of Environmental and Occupational Health, University of South Florida, College of Public Health Tampa, Florida, USA; Department of Epidemiology and Biostatistics, University of South Florida, College of Public Health Tampa, Florida, USA; and Center of Excellence in Health Equity, Training and Research, Baylor College of Medicine, Houston, TX, USA

\Corresponding author email: zenab.yusuf@bcm.edu

\section{ABSTRACT}

Background: Childhood obesity is one of the foremost threats to population health in the United States (U.S.) leading to the emergence of co-morbidities and increased healthcare cost. We explore the influence of selected social determinants of health (SDOH) on overweight and obesity among U.S. children.

Methods: We utilized the National Survey of Children's Health (NSCH) 2016-I7 dataset for this analysis. Overweight was defined as Body Mass Index $(B M I) \geq 85^{\text {th }}$ to $<95^{\text {th }}$, while obesity was defined as $\mathrm{BMI} \geq 95^{\text {th }}$ percentile for age and sex. Based on the literature and pathway plausibility, we examined several SDOH variables as predictors of childhood overweight or obesity in the US. Survey log-binomial regression models were built to generate prevalence ratio (PR) estimates to capture the associations between SDOH and overweight or obesity.

Results: About 30.6 million children were surveyed of which 9.5 million (31.0\%) were either overweight or obese. The likelihood of obesity was elevated among non-Hispanic Black and Hispanic children $(P R=I .53 ; 95 \% \mathrm{Cl}=1.0 \mathrm{I}-2.3 \mathrm{I})$ and $(\mathrm{PR}=\mathrm{I} .50 ; 95 \% \mathrm{Cl}=\mathrm{I} . \mathrm{I}$ 8- I.90) respectively. Overweight was more frequent in younger children, children of single parents, and children who lived in a neighborhood with no amenities. Parental attainment of college education, health insurance coverage, female gender, and language spoken in home other than Spanish were protective against overweight or obesity.

Conclusions and Global Health Implications: SDOH represent markers of overweight or obesity in children. We recommend the development of innovative interventions using SDOH risk and protective pathways as guide to address the current epidemic of childhood overweight and obesity.

Key words: Social determinants • Obesity • Overweight $・$ SDOH $・$ Children • United States

Copyright ( 2020 Yusuf et al. Published by Global Health and Education Projects, Inc. This is an open-access article distributed under the terms of the Creative Commons Attribution License CC BY 4.0. 


\section{Introduction}

\section{I.I. Background}

Childhood obesity is one of the foremost threats to population health in the United States (U.S.)., Childhood obesity refers to a body mass index (BMI) at or greater than $95^{\text {th }}$ percentile for age and sex, while childhood overweight is BMI at or greater than $85^{\text {th }}$ to less than $95^{\text {th }}$ percentile for age and sex. ${ }^{3}$ Globally, the mean standardized BMI increased by $0.32 \mathrm{~kg} / \mathrm{m}^{2}$ per decade between 1975 and $2016 .{ }^{4}$ In the U.S. and over the last three decades, the prevalence of childhood obesity has increased by more than four-folds. ${ }^{5}$ Mean obesity prevalence in children 6 -II years of age increased from $4 \%$ to 18.4\%; and in children $12-19$ years from $6.1 \%$ to $20.6 \%$ between 1971 to $1974,5,6$ and between 2015 to 2016,7 respectively. The rising prevalence of childhood overweight and obesity is associated with the emergence of comorbidities such as obstructive sleep apnea, type 2 diabetes mellitus, dyslipidemia, hypertension, and non-alcoholic fatty liver disease. ${ }^{3}$ These comorbidities are positively correlated with the severity of obesity ${ }^{3}$ and do influence adult life morbidity and mortality.' Additionally, psychosocial problems such as lowered self-esteem, depression, and peer discrimination are experienced by overweight and obese children. ${ }^{8}$ Furthermore, there are significant societal and economic burdens associated with the rising prevalence of childhood obesity and related comorbidities. " The dire consequences associated with childhood obesity which progress into adulthood include poor academic and lower skill achievements, inferior outcomes in the labor market, rising healthcare costs, and increasing decline in productivity. ${ }^{9}$

Several studies have assessed the independent relationships between sociodemographic and economic factors on childhood obesity in the U.S. particularly, the influence of age, gender, race/ethnicity and socioeconomic status on childhood obesity. . $^{1015}$ There are, however, fewer studies examining the association of selected social determinants such as socioeconomic, behavioral, and environmental factors on childhood overweight and obesity in the U.S. ${ }^{5}$

\section{I.2. Objective}

The aim of this study was to explore the association of selected social determinants of health (SDOH) comprising socioeconomic, behavioral, and environmental factors on overweight and obesity among children in the U.S. using nationally representative data.

\section{Methods}

\section{I. Study Population/variables}

This study utilized survey data from the National Survey of Children's Health (NSCH) 2016-17.16 The NSCH is sponsored and directed by the Health Resources and Services Administration (HRSA) Maternal and Child Health Bureau (MCHB). ${ }^{16}$ Beginning 2016, NSCH started integrating two surveys, the previous NSCH and the National Survey of Children with Special Health Care Needs (NS$\mathrm{CSHCN}$ ), which were administered via web and mail (paper) instruments. The majority of the questions in the NSCH were the same in both the 2016 and 2017 surveys. Only items that were the same across both data sets were included in the combined dataset for this analysis. From 2016 onward, the $\mathrm{NSCH}$ is being conducted annually by the U.S. Census Bureau, and the 2017 survey was the second administration of the redesigned NSCH.The survey provides rich data on multiple factors of health and well-being (physical, emotional, and mental), access to quality health care, neighborhood, school, and social context of noninstitutionalized children 0-17 years of age living in the US.

\section{I.I. Sampling strategy}

Households with one or more children under 18 years old were randomly sampled and contacted by mail, and one child from each household was randomly selected to be the subject of the survey. Then an adult (usually a parent or guardian) in the household who was most familiar with the child's health and health care was invited to complete a short screener questionnaire and a more detailed age-specific topical questionnaire online or by paper. Children with special health care needs and children 0-5 years of age were oversampled in the survey. A total of 7I,8II surveys were completed for 2016 
and 2017 combined comprising 50,212 surveys in 2016 and 21,599 in 2017. Survey data were weighted and adjusted for the combined dataset to represent the population of noninstitutionalized children aged 0-I7 years living in housing units nationally and in each state. The overall weighted response rate was $40.7 \%$ for 2016 and $37.4 \%$ for 2017 . The survey data was weighted to allow for generalizations to state and national pediatric populations (Screener and Topical file), and households with children (Screener file). The final weight for selected children, household, and child screener weights were assigned to all households and children with completed screeners. ${ }^{16}$

\section{I.2. Exposure and outcome variables}

Our exposures of interest were selected social determinants of health (SDOH) comprising demographic, socioeconomic, behavioral, and environmental characteristics. Demographic factors included sex categorized as male and female, age sub-grouped into $10-12$ years, 13 - 15 years, and 16 - 17 years; and race/ethnicity categorized into non-Hispanic White, non-Hispanic Black, Hispanic and Other. Socioeconomic factors included parents' nativity categorized into parent(s) born in the US, any parent born outside the US, other (child born in the US, parents not listed); primary household language categorized into Hispanic children with non-English primary household language, Hispanic children with English as primary household language and nonHispanic children; family structure categorized as two parents currently married, two parents who are not currently married, single parent, other family member with no parent reported; poverty level had 4 subgroups based on Federal Poverty Level (FPL) which were 0 - $99 \%$ FPL, 100 - 199\% FPL, $200-399 \%$ FPL and $400 \% \mathrm{FPL}$ and above; highest education attained by the adults in the household categorized as less than high school, high school degree or GED, some college or technical school and college degree or higher; insurance type classified as public only, private only, public and private insurance and currently uninsured. Behavioral characteristic was measured by how well the parents were able to cope with day to day demands of raising children categorized as "very well", "somewhat well" and "not very well or not very well at all". Environmental characteristic was measured as the number of neighborhood amenities such as: (I) sidewalks or walking paths; (2) park or playground area;(3) recreation center, community center or Boys'/Girls' Club; (4) library or bookmobile the child had access to. The response options were no amenities, I amenity, 2 amenities, 3 amenities and 4 amenities.

The outcomes of interest were overweight and obesity in children 10 to 17 years. As reported in previous studies that parents typically overestimate height and underestimate weight of children younger than 10 years of age, ${ }^{28}$ the BMI for children under the age of 10 years was therefore, not reported in the survey.

\subsection{Statistical Analysis}

We conducted weighted bivariate analysis using Pearson's Chi-squared test to determine the association between various exposure variables and overweight or obesity. To adjust for confounders, we ran weighted survey log binomial logistic regression which generated prevalence ratio (PR) estimates for the association between our exposure and outcome variables. We constructed three models separately for (I) overweight only; (2) obese only; and (3) overweight or obese. All tests of hypotheses were two-tailed with a type I error fixed at 5\%.

\subsection{Ethical Approval}

This study was approved as exempt by the Institutional Review Board of Baylor College of Medicine.

\section{Results}

A total of 30.6 million children aged $0-17$ years were surveyed. Of these, 9.5 million children aged 10 17 years were either overweight or obese (31.0\%). The prevalence of children classified as overweight only, and obese only was $15.19 \%$ and $15.77 \%$ respectively.

\section{I. Sociodemographic Characteristics}

Table I shows overall distribution and prevalence of demographic, socioeconomic, and environmental characteristics comparing children that were 
Table I. Socio-demographic characteristics of U.S. children I0-I7 years according to non-overweight and overweight or obesity status

\begin{tabular}{|c|c|c|c|c|c|c|}
\hline Characteristics & $\begin{array}{c}\text { Non-over } \\
\text { weight } \\
\left(B M I<85^{\text {th }}\right) \\
\text { n=2 I I5949I }\end{array}$ & $\begin{array}{c}\text { Proportion } \\
\%=69.0\end{array}$ & $\begin{array}{c}\text { Overweight or } \\
\text { obesity } \\
\left(B M I 5^{\text {th }} \text { to } \geq 95^{\text {th }}\right) \\
n=9485994\end{array}$ & $\begin{array}{l}\text { Proportion } \\
\%=31.0\end{array}$ & p-value & Prevalence ${ }^{\#}$ \\
\hline \multicolumn{7}{|l|}{ Demographic characteristics } \\
\hline Sex & & & & & $<0.0001$ & \\
\hline Male & 10556223 & $49.9 \%$ & 5107233 & $53.8 \%$ & & $32.6 \%$ \\
\hline Female & 10603268 & $50.1 \%$ & 4378761 & $46.2 \%$ & & $29.2 \%$ \\
\hline Age & & & & & $<0.0001$ & \\
\hline $10-12$ years & 7377725 & $34.9 \%$ & 3832750 & $40.4 \%$ & & $34.2 \%$ \\
\hline $13-15$ years & 8198336 & $38.7 \%$ & 3502254 & $36.9 \%$ & & $29.9 \%$ \\
\hline 16-17 years & 5583430 & $26.4 \%$ & 2150990 & $22.7 \%$ & & $27.8 \%$ \\
\hline Race/ethnicity & & & & & $<0.0001$ & \\
\hline NH-White & 11926308 & $56.4 \%$ & 4109161 & $43.3 \%$ & & $25.6 \%$ \\
\hline $\mathrm{NH}$-Black & 2516748 & $11.9 \%$ & 1681717 & $17.7 \%$ & & $40.1 \%$ \\
\hline Hispanic & 4558876 & $21.5 \%$ & 2863638 & $30.2 \%$ & & $38.6 \%$ \\
\hline Others & 2157559 & $10.2 \%$ & 831478 & $8.8 \%$ & & $27.8 \%$ \\
\hline \multicolumn{7}{|l|}{ Socioeconomic characteristics } \\
\hline Parents' nativity & & & & & $<0.0001$ & \\
\hline $\begin{array}{l}\text { Parent (s) born in US } \\
\text { (at least one parent) }\end{array}$ & 14435674 & $68.2 \%$ & 5968120 & $62.9 \%$ & & $29.3 \%$ \\
\hline $\begin{array}{l}\text { Any parent born outside US } \\
\text { (at least one parent) }\end{array}$ & 5372948 & $25.4 \%$ & 2579730 & $27.2 \%$ & & $32.4 \%$ \\
\hline $\begin{array}{l}\text { Other (child born in U.S, parents } \\
\text { not listed) }\end{array}$ & 1137829 & $5.4 \%$ & 802192 & $8.5 \%$ & & $41.3 \%$ \\
\hline Primary household language (PHHL) & & & & & $<0.0001$ & \\
\hline Hispanic children, non-English PHHL & 1811395 & $8.6 \%$ & 1171059 & $12.3 \%$ & & $39.3 \%$ \\
\hline Hispanic children, English PHHL & 2656967 & $12.6 \%$ & 1613537 & $17.0 \%$ & & $37.8 \%$ \\
\hline Non-Hispanic child & 16520820 & $78.1 \%$ & 6568862 & $69.2 \%$ & & $28.4 \%$ \\
\hline Family structure & & & & & $<0.0001$ & \\
\hline Two parents currently married & |470278| & $69.5 \%$ & 5466241 & $57.6 \%$ & & $27.1 \%$ \\
\hline Two parents, not currently married & 1408492 & $6.7 \%$ & 755553 & $8.0 \%$ & & $34.9 \%$ \\
\hline $\begin{array}{l}\text { Single parent } \\
\text { (mother or father) }\end{array}$ & 3061135 & $14.5 \%$ & 1991738 & $21.0 \%$ & & $39.4 \%$ \\
\hline $\begin{array}{l}\text { Other family type, no parent } \\
\text { reported }\end{array}$ & 1720667 & $8.1 \%$ & 1094497 & $11.5 \%$ & & $38.9 \%$ \\
\hline Poverty level & & & & & $<0.0001$ & \\
\hline $0-99 \% \mathrm{FPL}$ & 3636078 & $17.2 \%$ & 2331532 & $24.6 \%$ & & $39.1 \%$ \\
\hline $100 \%-199 \% \mathrm{FPL}$ & 4117300 & $19.5 \%$ & 2263631 & $23.9 \%$ & & $35.5 \%$ \\
\hline $200 \%-399 \%$ FPL & 5709372 & $27.0 \%$ & 2629827 & $27.7 \%$ & & $31.5 \%$ \\
\hline $400 \%$ FPL or above & 7696741 & $36.4 \%$ & 2261004 & $23.8 \%$ & & $22.7 \%$ \\
\hline Highest education of household adult & & & & & $<0.0001$ & \\
\hline Less than high school & 1763797 & $8.3 \%$ & 1321308 & $13.9 \%$ & & $42.8 \%$ \\
\hline
\end{tabular}

(Contd...) 
Table I. (Continued)

\begin{tabular}{|c|c|c|c|c|c|c|}
\hline Characteristics & $\begin{array}{c}\text { Non-over } \\
\text { weight } \\
\left(\mathrm{BMI}<85^{\text {th }}\right) \\
\mathrm{n}=2 \text { I I5949I }\end{array}$ & $\begin{array}{c}\text { Proportion } \\
\%=69.0\end{array}$ & $\begin{array}{c}\text { Overweight or } \\
\text { obesity } \\
\left(B M \mid 85^{\text {th }} \text { to } \geq 95^{\text {th }}\right) \\
n=9485994\end{array}$ & $\begin{array}{l}\text { Proportion } \\
\%=31.0\end{array}$ & p-value & Prevalence ${ }^{\#}$ \\
\hline High school degree or GED & 3732854 & $17.6 \%$ & 2394485 & $25.2 \%$ & & $39.1 \%$ \\
\hline Some college or technical school & 4623420 & $21.9 \%$ & 2512813 & $26.5 \%$ & & $35.2 \%$ \\
\hline College degree or higher & 10897275 & $51.5 \%$ & 3186337 & $33.6 \%$ & & $22.6 \%$ \\
\hline Insurance type & & & & \multicolumn{3}{|c|}{$<0.0001$} \\
\hline Public only & 5007232 & $23.7 \%$ & 3460596 & $36.5 \%$ & & $40.9 \%$ \\
\hline Private only & I3543873 & $64.0 \%$ & 4520148 & $47.7 \%$ & & $25.0 \%$ \\
\hline Currently uninsured & I305664 & $6.2 \%$ & 710410 & $7.5 \%$ & & $35.2 \%$ \\
\hline Public and private insurance & 888280 & $4.2 \%$ & 572593 & $6.0 \%$ & & $39.2 \%$ \\
\hline \multicolumn{7}{|l|}{ Behavioral characteristic } \\
\hline $\begin{array}{l}\text { Parents' coping with day to day } \\
\text { demands of raising children }\end{array}$ & & & & \multicolumn{3}{|c|}{$<0.0001$} \\
\hline Very well & 13821997 & $65.3 \%$ & 6267432 & $66.1 \%$ & & $31.2 \%$ \\
\hline Somewhat well & 6833958 & $32.3 \%$ & 2979310 & $31.4 \%$ & & $30.4 \%$ \\
\hline Not very well or not very well at all & $2988 \mid 4$ & $1.4 \%$ & 122042 & $1.3 \%$ & & $29.0 \%$ \\
\hline \multicolumn{7}{|l|}{ Environmental characteristic } \\
\hline Neighborhood amenities* & & & & \multicolumn{3}{|c|}{$<0.0001$} \\
\hline $\begin{array}{l}\text { Neighborhood does not contain } \\
\text { any amenities }\end{array}$ & 2250267 & $10.6 \%$ & II 55408 & $12.2 \%$ & & $33.9 \%$ \\
\hline Neighborhood contains I amenity & 2075021 & $9.8 \%$ & II 22736 & $11.8 \%$ & & $35.1 \%$ \\
\hline Neighborhood contains 2 amenities & 3300468 & $15.6 \%$ & 1362376 & $14.4 \%$ & & $29.2 \%$ \\
\hline Neighborhood contains 3 amenities & 4675674 & $22.1 \%$ & 2127837 & $22.4 \%$ & & $31.3 \%$ \\
\hline $\begin{array}{l}\text { Neighborhood contains all } 4 \\
\text { amenities }\end{array}$ & 8134344 & $38.4 \%$ & 3343060 & $35.2 \%$ & & $29.1 \%$ \\
\hline
\end{tabular}

Total of some variables not $100 \%$ because missing values were excluded.

* Neighborhood amenities are I) sidewalks or walking paths, 2) park or playground area, 3) recreation center, community center or Boys'/Girls' Club, 4) library or bookmobile

\# Prevalence of overweight/obesity

overweight or obese versus those that were non-overweight. Chi-square test revealed statistically significant differences $(p<0.000 \mathrm{I})$ across all selected variables. Children that were overweight or obese were more likely to be boys (53.8\%), in the I0- 12 years age group (40.4\%), Hispanic (30.2\%), living with a single parent and more likely to be living with an adult with high school degree or GED (25.2\%) compared to their non-overweight counterparts. There was a dose- response relationship observed between obesity and poverty level with rising prevalence of obesity/overweight with incremental poverty levels $(\mathrm{P}<0.000 \mathrm{I})$.

Table 2 presents the characteristics of children who were overweight only or obese only.
Compared to those who were overweight, obese children were more likely to be Hispanic (22.5\%), to speak a language other than English at home (23.8\%), to live with a single parent $(21.6 \%)$, and to come from a poorer household (22.9\%). Obese children were also more likely to have a parent with less than high school educational attainment (25.0 $\%$ versus $17.8 \%$ ).

\subsection{Prevalence of Overweight and Obesity}

Table 3 provides the estimates for the association between $\mathrm{SDOH}$ and overweight or obesity, overweight only, and obesity only. The probability of overweight or obesity was higher in children 10-12 years 34\% $(95 \% \mathrm{Cl}=16 \%-56 \%)$ and $13-15$ years $12 \%(95 \% \mathrm{Cl}$ 
Overweight and Obesity in U.S. Children

Table 2. Weighted prevalence (\%) of overweight or obesity among U.S. children aged I0-I7 years

\begin{tabular}{|c|c|c|c|c|c|c|}
\hline Characteristics & $\begin{array}{c}\text { Overweight } \\
\text { only }\end{array}$ & $\begin{array}{c}\text { Overweight } \\
\text { prevalence } \\
(15.2 \%)\end{array}$ & p-value & $\begin{array}{c}\text { Obesity } \\
\text { only }\end{array}$ & $\begin{array}{c}\text { Obesity } \\
\text { prevalence (15.8\%) }\end{array}$ & p-value \\
\hline \multicolumn{7}{|l|}{ Demographic characteristics } \\
\hline Sex & & & $<0.0001$ & & & $<0.0001$ \\
\hline Male & $49.1 \%$ & $14.6 \%$ & & $58.4 \%$ & $18.0 \%$ & \\
\hline Female & $50.9 \%$ & $15.8 \%$ & & $41.6 \%$ & $13.4 \%$ & \\
\hline Age & & & $<0.0001$ & & & $<0.0001$ \\
\hline $10-12$ years & $40.1 \%$ & $16.6 \%$ & & $40.7 \%$ & $17.5 \%$ & \\
\hline $13-15$ years & $39.6 \%$ & $15.8 \%$ & & $34.3 \%$ & $14.2 \%$ & \\
\hline $16-17$ years & $20.3 \%$ & $12.2 \%$ & & $25.0 \%$ & $15.6 \%$ & \\
\hline Race/ethnicity & & & $<0.0001$ & & & $<0.0001$ \\
\hline NH-White & $28.6 \%$ & $17.9 \%$ & & $31.7 \%$ & $20.6 \%$ & \\
\hline $\mathrm{NH}$-Black & $45.3 \%$ & $13.2 \%$ & & $41.4 \%$ & $12.5 \%$ & \\
\hline Hispanics & $15.9 \%$ & $17.6 \%$ & & $19.5 \%$ & $22.5 \%$ & \\
\hline Others & $10.2 \%$ & $15.9 \%$ & & $7.4 \%$ & $11.9 \%$ & \\
\hline \multicolumn{7}{|l|}{ Socioeconomic characteristics } \\
\hline Parents' nativity & & & $<0.0001$ & & & $<0.0001$ \\
\hline Parent (s) born in US & $63.7 \%$ & $14.5 \%$ & & $62.2 \%$ & $14.7 \%$ & \\
\hline Any parent born outside US & $27.2 \%$ & $15.9 \%$ & & $27.1 \%$ & $16.5 \%$ & \\
\hline $\begin{array}{l}\text { Other (child born in U.S, } \\
\text { parents not listed) }\end{array}$ & $7.7 \%$ & $18.5 \%$ & & $9.2 \%$ & $22.9 \%$ & \\
\hline $\begin{array}{l}\text { Primary household } \\
\text { language (PHHL) }\end{array}$ & & & $<0.0001$ & & & $<0.0001$ \\
\hline $\begin{array}{l}\text { Hispanic children, non-English } \\
\text { PHHL }\end{array}$ & $9.9 \%$ & $15.4 \%$ & & $14.7 \%$ & $23.8 \%$ & \\
\hline $\begin{array}{l}\text { Hispanic children, English } \\
\text { PHHL }\end{array}$ & $17.8 \%$ & $19.4 \%$ & & $16.3 \%$ & $18.4 \%$ & \\
\hline Non-Hispanic child & $70.9 \%$ & $14.3 \%$ & & $67.7 \%$ & $14.2 \%$ & \\
\hline Family structure & & & $<0.0001$ & & & $<0.0001$ \\
\hline $\begin{array}{l}\text { Two parents, currently } \\
\text { married }\end{array}$ & $60.0 \%$ & $13.8 \%$ & & $55.4 \%$ & $13.3 \%$ & \\
\hline $\begin{array}{l}\text { Two parents, not currently } \\
\text { married }\end{array}$ & $8.0 \%$ & $17.2 \%$ & & $7.9 \%$ & $17.7 \%$ & \\
\hline $\begin{array}{l}\text { Single parent } \\
\text { (mother or father) }\end{array}$ & $19.3 \%$ & $17.8 \%$ & & $22.6 \%$ & $21.6 \%$ & \\
\hline $\begin{array}{l}\text { Other family type, no parent } \\
\text { reported }\end{array}$ & $11.2 \%$ & $18.6 \%$ & & $11.8 \%$ & $20.3 \%$ & \\
\hline Poverty level & & & $<0.0001$ & & & $<0.0001$ \\
\hline 0-99\% FPL & $20.7 \%$ & $16.2 \%$ & & $28.3 \%$ & $22.9 \%$ & \\
\hline $100 \%-199 \% \mathrm{FPL}$ & $22.4 \%$ & $16.3 \%$ & & $25.3 \%$ & $19.1 \%$ & \\
\hline 200\%-399\% FPL & $28.1 \%$ & $15.7 \%$ & & $27.3 \%$ & $15.8 \%$ & \\
\hline $400 \%$ FPL or above & $28.7 \%$ & $13.4 \%$ & & $19.1 \%$ & $9.3 \%$ & \\
\hline
\end{tabular}




\section{Table 2: (Continued)}

\begin{tabular}{|c|c|c|c|c|c|c|}
\hline Characteristics & $\begin{array}{l}\text { Overweight } \\
\text { only }\end{array}$ & $\begin{array}{l}\text { Overweight } \\
\text { prevalence } \\
(15.2 \%)\end{array}$ & p-value & $\begin{array}{l}\text { Obesity } \\
\text { only }\end{array}$ & $\begin{array}{c}\text { Obesity } \\
\text { prevalence (15.8\%) }\end{array}$ & p-value \\
\hline $\begin{array}{l}\text { Highest education of } \\
\text { household adult }\end{array}$ & & & $<0.0001$ & & & $<0.0001$ \\
\hline Less than high school & $11.8 \%$ & $17.8 \%$ & & $16.0 \%$ & $25.0 \%$ & \\
\hline High school degree or GED & $22.5 \%$ & $17.1 \%$ & & $27.9 \%$ & $22.0 \%$ & \\
\hline $\begin{array}{l}\text { Some college or technical } \\
\text { school }\end{array}$ & $25.7 \%$ & $16.8 \%$ & & $27.3 \%$ & $18.5 \%$ & \\
\hline College degree or higher & $39.3 \%$ & $13.0 \%$ & & $28.1 \%$ & $9.6 \%$ & \\
\hline Insurance type & & & $<0.0001$ & & & $<0.0001$ \\
\hline Public only & $32.8 \%$ & $18.0 \%$ & & $40.0 \%$ & $22.8 \%$ & \\
\hline Private only & $53.8 \%$ & $13.9 \%$ & & $41.7 \%$ & $11.2 \%$ & \\
\hline Public and private insurance & $3.4 \%$ & $10.8 \%$ & & $8.6 \%$ & $28.4 \%$ & \\
\hline Currently uninsured & $7.7 \%$ & $17.7 \%$ & & $7.3 \%$ & $17.5 \%$ & \\
\hline \multicolumn{7}{|l|}{ Behavioral characteristic } \\
\hline $\begin{array}{l}\text { Parents' coping with day to day } \\
\text { demands of raising children }\end{array}$ & & & $<0.0001$ & & & $<0.0001$ \\
\hline Very well & $64.0 \%$ & $14.8 \%$ & & $68.1 \%$ & $16.4 \%$ & \\
\hline Somewhat well & $33.5 \%$ & $15.9 \%$ & & $29.4 \%$ & $14.5 \%$ & \\
\hline $\begin{array}{l}\text { Not very well or not very } \\
\text { well at all }\end{array}$ & $1.2 \%$ & $13.0 \%$ & & $1.4 \%$ & $16.0 \%$ & \\
\hline \multicolumn{7}{|l|}{ Environmental characteristic } \\
\hline Neighborhood amenities* & & & $<0.0001$ & & & $<0.0001$ \\
\hline $\begin{array}{l}\text { Neighborhood does not } \\
\text { contain any amenities }\end{array}$ & $12.7 \%$ & $17.4 \%$ & & $11.6 \%$ & $16.5 \%$ & \\
\hline $\begin{array}{l}\text { Neighborhood contains I } \\
\text { amenity }\end{array}$ & $11.3 \%$ & $16.5 \%$ & & $12.3 \%$ & $18.6 \%$ & \\
\hline $\begin{array}{l}\text { Neighborhood contains } 2 \\
\text { amenities }\end{array}$ & $14.6 \%$ & $14.6 \%$ & & $14.1 \%$ & $14.6 \%$ & \\
\hline $\begin{array}{l}\text { Neighborhood contains } 3 \\
\text { amenities }\end{array}$ & $22.0 \%$ & $15.0 \%$ & & $22.9 \%$ & $16.2 \%$ & \\
\hline $\begin{array}{l}\text { Neighborhood contains all } 4 \\
\text { amenities }\end{array}$ & $34.7 \%$ & $14.1 \%$ & & $35.7 \%$ & $15.0 \%$ & \\
\hline
\end{tabular}

Total of some variables not $100 \%$ because missing values were excluded.

* Neighborhood amenities are I) sidewalks or walking paths, 2) park or playground area, 3) recreation center, community center or Boys'/Girls' Club, 4) library or bookmobile

= 96\%-29\%)] respectively, using children $16-17$ years as referent category. Children who lived with a single parent had a greater probability of being overweight or obese $32 \%(95 \% \mathrm{Cl}=12 \%-55 \%)$ compared to those having two parents who were currently married. Similarly, compared to children who lived in neighborhoods containing all 4 amenities, those who lived in neighborhoods having one or less amenity, were $27 \%(95 \% \mathrm{Cl}=2 \%-58 \%)$ more likely to be overweight or obese. Conversely, factors protective of being overweight or obese included; children who lived with an adult with more than 12 years of education ( $P R=$ $0.57 ; 95 \% \mathrm{Cl}=0.42-0.79)$ and having private health insurance ( $P R=0.71 ; 95 \% \mathrm{Cl}=0.59-0.87$ ) (Table 3$)$.

\section{Prevalence of overweight only}

In the adjusted model for overweight only, compared to children 16-17 years, children 10-12 years and those $13-15$ years had a $45 \%(95 \% \mathrm{Cl}=20 \%-74 \%)$ and $35 \%(95 \% \mathrm{Cl}=11 \%-64 \%)$ higher probability of 
Table 3. Adjusted prevalence ratio model for social determinants of health in overweight or obesity, overweight only and obesity only among U.S. children aged I 0 - I 7 years

\begin{tabular}{|c|c|c|c|c|c|c|}
\hline Characteristics & $\begin{array}{c}\text { Overweight or } \\
\text { obese } \\
\text { adjusted PR }\end{array}$ & p-value & $\begin{array}{l}\text { Overweight } \\
\text { only } \\
\text { adjusted PR }\end{array}$ & p-value & Obese only & $\begin{array}{l}\text { Adjusted PR } \\
\text { P value }\end{array}$ \\
\hline \multicolumn{7}{|l|}{ Demographic characteristics } \\
\hline \multicolumn{7}{|l|}{ Sex } \\
\hline Male & Reference & & & & & \\
\hline Female & $0.88(0.78-0.99)$ & 0.15 & $\mathrm{I} .10(0.94-\mathrm{I} .27)$ & 0.23 & $0.74(0.63-0.87)$ & $<0.0001$ \\
\hline \multicolumn{7}{|l|}{ Age } \\
\hline $16-17$ years & Reference & & & & & \\
\hline $10-12$ years & $1.34(1.16-1.56)$ & $<0.0001$ & $\mathrm{I} .45(\mathrm{I} .20-\mathrm{I} .74)$ & $<0.0001$ & I.I2 (0.92-I.36) & 0.26 \\
\hline $13-15$ years & $1.12(0.96-1.29)$ & $<0.0001$ & $\mathrm{I} .35(\mathrm{I} . \mathrm{II}-\mathrm{I} .64)$ & $<0.0001$ & $0.89(0.73-1.08)$ & 0.25 \\
\hline \multicolumn{7}{|l|}{ Race/ethnicity } \\
\hline NH-White & Reference & & & & & \\
\hline $\mathrm{NH}-$ Black & 1.41 (1.0I-I.98) & 0.03 & I.I 2 (0.72-I.74) & 0.61 & $\mathrm{I} .53(\mathrm{I} .0 \mathrm{I}-2.3 \mathrm{I})$ & 0.04 \\
\hline Hispanic & $1.52(1.25-1.85)$ & 0.04 & $1.29(1.00-1.65)$ & 0.05 & $1.50(1.18-1.90)$ & $<0.0001$ \\
\hline Multiracial/NH-other & I.II (0.92-I.34) & $<0.0001$ & $1.29(1.03-1.62)$ & 0.03 & $0.89(0.69-I .14)$ & 0.34 \\
\hline \multicolumn{7}{|l|}{ Socioeconomic characteristics } \\
\hline \multicolumn{7}{|l|}{ Parents' nativity } \\
\hline Parent (s) born in US & Reference & & & & & \\
\hline Any parent born outside US & $0.90(0.74-1.09)$ & 0.27 & $\mathrm{I} .02(0.8 \mathrm{I}-\mathrm{I} .29)$ & 0.88 & $0.83(0.63-1.08)$ & 0.17 \\
\hline $\begin{array}{l}\text { Other (child born in U.S, parents are } \\
\text { not listed) }\end{array}$ & $1.08(0.7 \mid-1.65)$ & 0.29 & $0.80(0.46-I .4 I)$ & 0.45 & I.43 (0.87-2.36) & 0.16 \\
\hline \multicolumn{7}{|l|}{ Primary household language (PHHL) } \\
\hline Hispanic children, non-English PHHL & Reference & & & & & \\
\hline Hispanic children, English PHHL & $1.09(0.75-1.57)$ & 0.72 & $\mathrm{I} .32(0.82-2 . \mathrm{II})$ & 0.25 & $0.88(0.56-1.37)$ & 0.57 \\
\hline Non-Hispanic child & $0.56(0.51-0.63)$ & 0.45 & $0.88(0.61-1.26)$ & 0.49 & $0.53(0.38-0.74)$ & $<0.000$ I \\
\hline \multicolumn{7}{|l|}{ Family structure } \\
\hline Two parents currently married & Reference & & & & & \\
\hline Two parents not currently married & $\mathrm{I} .05(0.80-\mathrm{I} .37)$ & 0.74 & $\mathrm{I} .06(0.76-\mathrm{I} .48)$ & 0.73 & $\mathrm{I} .02(0.74-\mathrm{I} .40)$ & 0.92 \\
\hline Single parent (mother or father) & $1.32(1.12-1.55)$ & $<0.0001$ & $1.26(1.03-1.54)$ & 0.03 & I.22 (0.99-I.50) & 0.06 \\
\hline Other family type, no parent & I.II (0.8I-I.52) & 0.52 & $\mathrm{I} .45(0.93-2.25)$ & 0.1 & $0.78(0.54-I . I 3)$ & 0.19 \\
\hline \multicolumn{7}{|l|}{ Poverty level } \\
\hline 0-99\% FPL & Reference & & & & & \\
\hline $100 \%-199 \% \mathrm{FPL}$ & $\mathrm{I} .06(0.85-\mathrm{I} .3 \mathrm{I})$ & 0.62 & $\mathrm{I} .09(0.84-\mid .4 \mathrm{I})$ & 0.52 & I.0I (0.78-I.3I) & 0.93 \\
\hline $200 \%-399 \% \mathrm{FPL}$ & $1.22(0.97-\mid .53)$ & 0.09 & I.20 (0.88-I.64) & 0.25 & I.I5 (0.86-I.55) & 0.34 \\
\hline $400 \%$ FPL or above & $\mathrm{I} .02(0.8 \mathrm{I}-\mathrm{I} .27)$ & 0.89 & I.I 5 (0.86-I.55) & 0.34 & $0.85(0.64-1.13)$ & 0.27 \\
\hline \multicolumn{7}{|l|}{ Highest education of household adult } \\
\hline Less than high school & Reference & & & & & \\
\hline High school degree or GED & $0.98(0.7|-| .36)$ & 0.91 & $0.99(0.63-1.54)$ & 0.96 & $0.99(0.69-\mathrm{I} .43)$ & 0.97 \\
\hline Some college or technical school & $0.86(0.62-1.19)$ & 0.36 & $0.95(0.6 \mathrm{I}-\mathrm{I} .48)$ & 0.81 & $0.86(0.60-1.23)$ & 0.40 \\
\hline College degree or higher & $0.57(0.42-0.79)$ & $<0.0001$ & $0.77(0.50-1.20)$ & 0.25 & $0.52(0.37-0.75)$ & $<0.0001$ \\
\hline
\end{tabular}

(Contd...) 
Table 3: (Continued)

\begin{tabular}{|c|c|c|c|c|c|c|}
\hline Characteristics & $\begin{array}{c}\text { Overweight or } \\
\text { obese } \\
\text { adjusted PR }\end{array}$ & p-value & $\begin{array}{l}\text { Overweight } \\
\text { only } \\
\text { adjusted PR }\end{array}$ & p-value & Obese only & $\begin{array}{l}\text { Adjusted PR } \\
\text { P value }\end{array}$ \\
\hline \multicolumn{7}{|l|}{ Insurance type } \\
\hline Public only (government assistance) & Reference & & & & & \\
\hline Private only & $0.71(0.59-0.87)$ & $<0.0001$ & $0.91(0.72-1.17)$ & 0.47 & $0.65(0.50-0.84)$ & $<0.0001$ \\
\hline Public and private insurance & $0.95(0.68-1.33)$ & 0.75 & $0.59(0.4 \mid-0.85)$ & $<0.0001$ & I.3| (0.87-I.98) & 0.20 \\
\hline Currently uninsured & $0.78(0.57-1.08)$ & 0.14 & $\mathrm{I} .02(0.68-\mathrm{I} .55)$ & 0.91 & $0.68(0.46-1.01)$ & 0.06 \\
\hline \multicolumn{7}{|l|}{ Behavioral characteristic } \\
\hline \multicolumn{7}{|c|}{ Parents' coping with day to day demands of raising children } \\
\hline Very well & Reference & & & & & \\
\hline Somewhat well & $0.96(0.85-1.08)$ & 0.48 & $\mathrm{I} .07(0.9 \mathrm{I}-\mathrm{I} .26)$ & 0.4 & $0.87(0.75-1.02)$ & 0.08 \\
\hline Not very well or not very well at all & $0.85(0.56-1.27)$ & 0.42 & $0.98(0.63-1.52)$ & 0.92 & $0.79(0.49-1.26)$ & 0.32 \\
\hline \multicolumn{7}{|l|}{ Environmental characteristic } \\
\hline \multicolumn{7}{|l|}{ Neighborhood amenities* } \\
\hline $\begin{array}{l}\text { Neighborhood contains all } 4 \\
\text { amenities }\end{array}$ & Reference & & & & & \\
\hline $\begin{array}{l}\text { Neighborhood does not contain any } \\
\text { amenities }\end{array}$ & $1.20(1.00-1.45)$ & 0.05 & $1.32(1.03-1.69)$ & 0.03 & $\mathrm{I} .02(0.80-\mathrm{I} .28)$ & 0.89 \\
\hline Neighborhood contains I amenity & $1.27(1.02-1.58)$ & 0.03 & $1.22(0.91-1.65)$ & 0.18 & $1.20(0.90-1.59)$ & 0.21 \\
\hline Neighborhood contains 2 amenities & $1.00(0.84-1.19)$ & 0.97 & $\mathrm{I} .06(0.87-\mathrm{I} .30)$ & 0.57 & $0.95(0.75-I .2 I)$ & 0.69 \\
\hline Neighborhood contains 3 amenities & $\mathrm{I} .08(0.92-\mathrm{I} .26)$ & 0.36 & I.I $2(0.92-1.35)$ & 0.26 & I.0I (0.8I-I.25) & 0.94 \\
\hline
\end{tabular}

being overweight respectively. Likewise, compared to non-Hispanic Whites, the probability of overweight in Hispanic and multiracial children were $29 \%(95 \%$ $\mathrm{Cl}=0 \%-65 \%)$ and $29 \%(95 \% \mathrm{Cl}=3 \%-62 \%)$ greater, respectively. Additionally, children who lived with a single parent $(P R=1.26 ; 95 \% \mathrm{Cl}=1.03-1.54)$ compared to those who lived with two married parents, as well as children who lived in a neighborhood with no amenities (PR $=1.32 ; 95 \% \mathrm{Cl}=1.03-1.69)$ compared to children who lived in a neighborhood with all four amenities were also more likely to be overweight. Inversely, having private and public insurance coverage protected children from being overweight $(P R=0.59$; $95 \% \mathrm{Cl}=0.4 \mid-0.85)$ compared to children who had public insurance/government assistance only (Table 3).

Prevalence of obesity only

The likelihood of obesity was $53 \%$ and $50 \%$ greater among non-Hispanic Black and Hispanic children compared to theirWhite counterparts. The strongest protective factors against childhood obesity include female gender $(P R=0.74 ; 95 \% \mathrm{Cl}=0.63-0.87)$, non-Hispanic speaking primary household language $(\mathrm{PR}=0.53 ; 95 \% \mathrm{Cl}=0.38-0.74)$ compared to nonEnglish speaking Hispanic child, parental attainment of college education or higher ( $P R=0.52 ; 95 \%$ $\mathrm{Cl}=0.37-0.75)$ compared to having less than high school education, and having private insurance coverage only $(\mathrm{PR}=0.65 ; 95 \% \mathrm{Cl}=0.5-0.84)$ as compared to having public insurance only (Table 3 ).

\section{Discussion}

The aim of this study was to understand and detail the potential influence of SDOH on childhood and adolescent overweight and obesity using a nationally representative sample in the United States. Our study showed that $31 \%$ of children in our study population were either overweight or obese; this finding is in consonance with previous studies which reported that the prevalence of overweight or obesity was between 30.9 - 34.0\%. ${ }^{17,19}$ More specifically, the prevalence of overweight in our 
study was $15.2 \%$, depicting similar findings with previous studies. ${ }^{6,19,20}$ However, other studies ${ }^{18,21,22}$ showed that the prevalence of overweight was between 28.8-33.4\%. Furthermore, we established that the prevalence of obesity was $15.8 \%$, similar to previous findings ${ }^{5,17-23}$ indicating a leveling of obesity since 2007. It is worth to note that the prevalence of overweight and obesity varied between studies as a result of geographic variation, ${ }^{24}$ methodology, ${ }^{25}$ and differences in types of unit of analysis used (individual vs. state vs. national level). ${ }^{19}$

Our study found that selected sociodemographic and environmental factors were associated with a higher probability of being overweight or obese. Concerning demographic factors, we observed that being a female child predicted less likelihood of obesity; a finding consistent with some studies conducted prior to 2016..$^{5-7,23,25}$ However, contrary to our results, some other studies either reported no difference in obesity prevalence between the sexes ${ }^{17,23}$ or found females to be at increased risk for childhood obesity. ${ }^{14}$ In addition, in relation to age, our adjusted model showed that younger children (age groups 10 12 and I 3 - I 5 years) were significantly more likely to be overweight. However, it was interesting that we did not observe any association between obesity and age, in contrast to the findings of Skinner et al. ${ }^{18}$ and Ogden ${ }^{14}$ who reported that obesity significantly increased with age. Our observations of a strong association between overweight and age are concordant with findings reported in previous studies. $5,7,8$

The findings in this study showed that the SDOH that could possibly protect a child from being overweight or obese were consistent with those of previous studies specifically, language spoken in the home other than Spanish (obesity only), ${ }^{5,26}$ parental attainment of college degree or higher (overweight or obese), $5,14,20,25$ and the possession of private or public insurance coverage. ${ }^{25,26}$ These protective socioeconomic determinants may provide children from these families the resources and opportunity of attaining and maintaining healthier physical and mental life styles. An interesting finding was that we, however, did not find poverty level to be an associated determinant of overweight or obesity. This finding was contrary to earlier studies showing that high income was less predictive of overweight or obesity. ${ }^{1,522,26}$ To our knowledge, this is the first study to examine parent's nativity as well as a parent's ability to cope with day-to-day demands of raising children in association with the social determinants of overweight or obesity. However, our analysis did not portray these two SDOH factors to be statistically significant predictors. Our results in relation to environmental characteristics confirm previous studies that children living in neighborhoods with no access to sidewalks or walking paths were more predisposed to obesity or overweight. ${ }^{1,22,25}$

\section{Limitations}

An important limitation in our study is that the data collected by NSCH was cross-sectional in nature, and that limited our ability to establish a temporal relationship between our exposures and outcomes. Nonetheless, the findings provide important information regarding the role of SDOH on childhood obesity.

\section{Recommendation for Further Studies}

Further research to clarify the relationship between overweight/obesity and SDOH in children is needed. Since overweight and obesity are multi-factorial in origin, in order to impact and bring about a reduction in their prevalence, it is crucial to develop effective preventive strategies aimed at addressing the relevant SDOH. We recommend the development of innovative multilevel individualized- as well as community-customized interventions using SDOH risk and protective pathways as guide to address the current childhood overweight and obesity epidemic.

\section{Conclusion and Global Health Implications}

The results of our study evidently illustrate that demographic, socioeconomic, and environmental characteristics represent important determinants of overweight and obesity in U.S. children. Our study showed that due to possible effective changes at the individual level, initiatives by schools and other communities, at the state, national, and global levels, the prevalence of obesity and overweight in the U.S appeared to have plateaued in 2016 and 
2017. ${ }^{17,27,28}$ More work needs to be done as the social determinants of overweight and obesity in children have significant national and global implications relating to $\mathrm{racial} / \mathrm{ethnic}$ disparities, population-based policy development, funding allocation, and prevention of overweight and obesity in childhood and subsequently in adulthood.

\section{Compliance with Ethical Standards}

Conflicts of Interest: The authors declare that they have no conflicts of interest. Financial Disclosure: This was a non-funded research. Funding/Support: None. Ethics Approval: This study was approved as exempt by the Institutional Review Board of Baylor College of Medicine. Acknowledgements: None.

\section{References}

I. Wang Y. Cross-national comparison of childhood obesity: the epidemic and the relationship between obesity and socioeconomic status. International Journal of Epidemiology. 200 I;30(5): I I29-36.

2. Koplan JP, Liverman CT, Kraak VA, eds. Preventing Childhood Obesity: Health in the Balance. Washington (DC): National Academies Press; 2005.

3. Kumar S, Kelly AS. Review of childhood obesity: from epidemiology, etiology, and comorbidities to clinical assessment and treatment. In Mayo Clinic Proceedings. 2017;92(2):25I-265.

4. Abarca-Gómez L, Abdeen ZA, Hamid ZA, Abu-Rmeileh NM, Acosta-Cazares B, Acuin C, Adams RJ, Aekplakorn W, Afsana K, Aguilar-Salinas CA, Agyemang C. Worldwide trends in body-mass index, underweight, overweight, and obesity from 1975 to 2016: a pooled analysis of 2416 population-based measurement studies in 128 . 9 million children, adolescents, and adults. Lancet. 2017;390( 101 I3):2627-2642.

5. Singh GK, Kogan MD,Van Dyck PC,Siahpush M.Racial/ ethnic, socioeconomic, and behavioral determinants of childhood and adolescent obesity in the United States: analyzing independent and joint associations. Annals of Epidemiology. 2008; |8(9):682-695.

6. Ogden $\mathrm{CL}$, Flegal KM, Carroll MD, Johnson CL. Prevalence and trends in overweight among US children and adolescents, 1999-2000. The Journal of the American Medical Association. 2002;288(14): 1728-1732.

7. Hales CM, Fryar CD, Carroll MD, Freedman DS,
Ogden CL. Trends in obesity and severe obesity prevalence in US youth and adults by sex and age, 2007-2008 to 2015-2016. The Journal of the American Medical Association. 2018;319(16): 1723-1725.

8. Au N. The health care cost implications of overweight and obesity during childhood. Health Services Research. 2012;47(2):655-676.

9. Finkelstein EA, Ruhm CJ, Kosa KM. Economic causes and consequences of obesity. Annual Review of Public Health. 2005;26:239-257.

10. Miech RA, Kumanyika SK, Setler N, Link BG, Phelan JC, Chang VW. Trends in the association of poverty with overweight among US adolescents, 1971-2004. The Journal of the American Medical Association. 2006;295:2385-2393.

II. Goodman E, Slap GB, Huang B. The public health impact of socioeconomic status on adolescent depression and obesity. American Journal Public Health. 2003;93:1844-1850.

12. Goodman E. The role of socioeconomic status gradients in explaining differences in US adolescents' health. American Journal Public Health. 1999;89:15221528.

13. Gordon-Larsen P, Adair LS, Pokin BM. The relationship of ethnicity, socioeconomic factors, and overweight in US adolescents. Obesity Research. 2003; | I:| $21-129$.

14. Ogden CL, Fryar CD, Hales CM, Carroll MD, Aoki Y, Freedman DS. Differences in obesity prevalence by demographics and urbanization in US children and adolescents, 2013-2016. The Journal of the American Medical Association. 2018;319(23):2410-24I8.

15. Powell LM, Wada R, Krauss RC, Wang Y. Ethnic disparities in adolescent body mass index in the United States: the role of parental socioeconomic status and economic contextual factors. Social Science \& Medicine. 201 2;75(3):469-476.

16. National Survey of Children's Health. Child and Adolescent Health Measurement Initiative. Data Resource Center for Child and Adolescent Health supported by the U.S. Department of Health and Human Services, Health Resources and Services Administration (HRSA), Maternal and Child Health Bureau (MCHB). 2016-20I7. Available at: https:// www.childhealthdata.org/browse/survey. Accessed April 18,2019.

17. Wang Y, Beydoun MA. The obesity epidemic in the 
United States-gender, age, socioeconomic, racial/ ethnic, and geographic characteristics: a systematic review and meta-regression analysis. Epidemiologic Reviews. 2007;29(I):6-28.

18. Skinner AC, Perrin EM, Skelton JA. Prevalence of obesity and severe obesity in US children, 199920|4. Obesity. 20 |6;24(5): I I |6- I I 23.

19. Wang Y, Wang JQ. A comparison of international references for the assessment of child and adolescent overweight and obesity in different populations. European Journal of Clinical Nutrition. 2002;56(I0):973-982.

20. Carey FR, Singh GK, Brown III HS, Wilkinson, AV. Educational outcomes associated with childhood obesity in the United States: cross-sectional results from the 20II-20I2 National Survey of Children's Health. International Journal of Behavioral Nutrition and Physical Activity. 20 I ; I 2(I):S3 200 I;30(5): I I 29- I I 36.

21. Borrell LN, Graham L, Joseph SP. Associations of Neighborhood Safety and Neighborhood Support with Overweight and Obesity in US Children and Adolescents. Ethnicity \& Disease. 2016;26(4):469.

22. Singh GK, Kogan MD, van Dyck PC. Changes in State-Specific Childhood Obesity and Overweight Prevalence in the United States From 2003 to 2007. Archives of Pediatrics \& Adolescent Medicine. 2010;164(7):598-607
23. Ogden CL, Carroll MD, Kit BK, Flegal KM. Prevalence of obesity and trends in body mass index among US children and adolescents, 1999-20I0. The Journal of the American Medical Association. 2012; 307(5):483-490.

24. Guedes DP,Rocha GD,SilvaAJ, Carvalhal IM, Coelho EM. Effects of social and environmental determinants on overweight and obesity among Brazilian schoolchildren from a developing region. Revista Panamericana de Salud Pública. 201 I;30(4):295-302.

25. Grow HM, Cook AJ, Arterburn DE, Saelens BE, Drewnowski A, Lozano P. Child obesity associated with social disadvantage of children's neighborhoods. Social Sciences \& Medicine. 20 10;7I (3):584-59I.

26. Flores G, Tomany-Korman SC. The language spoken at home and disparities in medical and dental health, access to care, and use of services in US children. Pediatrics. 2008; I 2 1(6): e 1703-17| 4.

27. Doak CM, Visscher TL, Renders CM, Seidell JC. The prevention of overweight and obesity in children and adolescents: a review of interventions and programmes. Obesity Reviews. 2006;7( I): I I I-136.

28. Long MW,Ward ZJ, Resch SC, CradockAL, Wang YC, Giles CM, Gortmaker SL. State-level estimates of childhood obesity prevalence in the United States corrected for report bias. International Journal of Obesity. 2016;40(10):1523. 